\title{
FATORES QUE POSSIBILITAM A REDUÇÃO DA DOSE DOS HERBICIDAS INIBIDORES DA ENZIMA ALS: REVISÃO DE LITERATURA
}

\begin{abstract}
O objetivo desta revisão de literatura foi organizar o conhecimento disponível na literatura com respeito aos fatores ambientais e fisiológicos que afetam a eficácia dos herbicidas inibidores de ALS. Dentre os fatores ambientais que afetam a eficácia dos herbicidas inibidores de ALS incluem-se a temperatura, a umidade relativa do ar, a irradiância e o estado nutricional das plantas. Dentre os fatores fisiológicos que afetam a dose empregada destacam-se as enzimas envolvidas na detoxificação dos herbicidas e as enzimas que aliviam os efeitos dos estresses oxidativos que decorrem da ação herbicida. Há necessidade de se investir em mais estudos integradores do conhecimento de forma a se desenvolver estratégias para otimizar a atividade dos herbicidas inibidores da ALS que possibilitem a redução da dose dos produtos sem comprometer a eficácia no controle das infestantes e, por consequência, garantir a produtividade das lavouras.
\end{abstract}

* Engenheiro Agrônomo, Mestrando do Programa de Pós-Graduação em Fitotecnia, Departamento de Plantas de Lavoura, Universidade Federal do Rio Grande do Sul (UFRGS), Porto Alegre, RS (e-mail: andrew_rerison@hotmail.com).

** Engenheiro Agrônomo, Ph. D., Professor, UFRGS, Pesquisador do CNPQ, Porto Alegre, RS (e-mail: ribas.vidal@gmail.com).

**** Engenheiro Agrônomo, Ph. D., Professor, UFRGS, Porto Alegre, RS (e-mail: merotto@ufrgs.br). 


\section{INTRODUÇÃO}

Os herbicidas inibidores da enzima acetolactato sintase (ALS), também conhecida como acetohidroxi ácido sintase (AHAS), são amplamente utilizados na agricultura brasileira. Trata-se de enzima chave na biossíntese dos aminoácidos essenciais de cadeia ramificada, valina, leucina e isoleucina (JURSíK, SOUKUP e HOLEC, 2010). Compartilham esse mecanismo de ação os herbicidas de diversos grupos químicos, incluindo imidazolinonas, sulfonilureias, triazolopirimidinas, pirimidinil-tiobenzoatos e sulfonil-amino-triazolinona, sendo os quatro primeiros utilizados no Brasil (CARVALHO, 2013). Mais de 55 moléculas da enzima ALS são comercializadas no mundo, das quais a maior parte é composta pelo grupo das sulfonilureias, com 34 ingredientes ativos registrados (HEAP, 2013). No Brasil, esses herbicidas são registrados pelo Sistema de Agrotóxicos Fitossanitários (AGROFIT) do Ministério da Agricultura, Pecuária e Abastecimento (MAPA) para culturas agrícolas de grande importância (BRASIL, 2013). Os herbicidas inibidores da ALS representam $31 \%$ (arroz); 24 \% (cana-de-açúcar); 14 \% (soja); 13 \% (milho); 11 \% (feijão) e 6 \% (algodão) dos ingredientes ativos registrados. Além disto, constituem $50 \%$ (aveia) e $12 \%$ (trigo) dos produtos disponíveis para o controle de plantas daninhas em culturas de inverno, com maior relevância na região Sul (BRASIL, 2013).

Entre os principais benefícios dos inibidores da enzima ALS destacam-se a elevada eficácia no controle de amplo espectro de espécies e o fato de que esses herbicidas são utilizados em baixas doses (gramas por hectare), em oposição às de diversos outros produtos (da ordem de quilogramas por hectare) (TRANEL e WRIGHT, 2002). Todavia, há possibilidade de reduzir a dose efetivamente utilizada pelo agricultor para evitar efeitos colaterais indesejados, tais como a contaminação do solo, a lixiviação para os lençóis freáticos e o desenvolvimento de populações vegetais resistentes aos herbicidas (MADAFIGLIO et al., 2000; YU et al., 2004; KIM et al., 2006; SØNDERSKOV, SWANTON e KUDSK, 2012).

A redução da dose dos herbicidas não pode comprometer a eficácia no controle das plantas daninhas, sob pena de que as plantas remanescentes prejudiquem a produtividade das culturas.

O objetivo desta revisão de literatura foi organizar o conhecimento disponível na literatura com respeito aos fatores ambientais e fisiológicos que afetam a eficácia dos herbicidas inibidores de ALS.

\section{EFEITO DA TEMPERATURA SOBRE A EFICÁCIA DOS INIBIDORES DA ALS}

Teoriza-se que as temperaturas cardeais (mínima, ótima e máxima) afetam a eficácia dos inibidores da ALS. Assim, para ampla faixa de temperaturas testadas, a eficácia dos herbicidas deve apresentar resposta quadrática. Todavia, a maioria dos experimentos para avaliar o impacto desse fator ambiental sobre a eficácia de herbicidas envolve faixa limitada de temperatura de forma que captam apenas parte da resposta esperada (POLGE \& BARRET, 1997; OLSON et al., 2000; WILLINGHAM et al., 2008). Por exemplo, a eficácia do herbicida imazaquim sobre a redução da massa seca de plantas de milho foi diretamente proporcional ao aumento da temperatura quando a faixa variou entre $18 / 12$ e $30 / 24^{\circ} \mathrm{C}$ (dia/noite) (POLGE \& BARRET, 1997). Efeito similar foi verificado para o herbicida sulfossulfurom aplicado em Aegilops cylindrica, Bromus secalinus L. e B. tectorum L. quando mantidas em condições de temperatura entre $5 / 3$ e $25 / 23{ }^{\circ} \mathrm{C}$ (OLSON et al., 2000). Todavia, em faixa de temperatura situada entre $21 / 11$ e $30 / 25^{\circ} \mathrm{C}$ (dia/noite), a eficácia do herbicida penoxsulam sobre o controle de Alternanthera philoxeroides mostrou-se inversamente proporcional à temperatura (WILLINGHAM et al., 2008).

Alguns fatores podem afetar a relação entre a eficácia e a temperatura, tais como a espécie vegetal avaliada, a taxa de detoxificação e o herbicida utilizado (GEIER, STAHLMAN e HARGETT 1999; KOEPPE et al., 2000; MCCULLOUGH e HART, 2006). O efeito da espécie pode ser ilustrado em experimentos realizados com o herbicida sulfossulfurom (MON 37500- inibidor da ALS seletivo 
para trigo) no controle de $B$. secalinus em Triticum aestivum (trigo), utilizando duas doses (9 e $18 \mathrm{~g} \mathrm{ha}^{-1}$ ) e o tratamento controle. Nos intervalos de temperatura de $10 / 5$ a $21 / 7{ }^{\circ} \mathrm{C}$ (dia/noite) foi evidenciada diferença na fitointoxicação causada pelo herbicida nas duas espécies, quando comparada à ação sob diversas temperaturas. Quando aspergido em $B$. secalinus, na dose de $9 \mathrm{~g} \mathrm{ha}^{-1}$, na faixa de temperatura de $10 / 5^{\circ} \mathrm{C}$ foi constatada maior fitotoxicidade em contraste ao efeito na temperatura de $21 / 7^{\circ} \mathrm{C}$, com redução de $72 \%$ e $61 \%$ da massa seca, respectivamente, em comparação ao tratamento sem aplicação do produto. No entanto, ambas as doses de sulfossulfurom em $T$. aestivum foram, consecutivamente, 8 a $13 \%$ mais fitotóxicas na faixa de temperatura mais elevada $\left(21 / 7^{\circ} \mathrm{C}\right)$ (GEIER, STAHLMAN e HARGETT, 1999).

Os herbicidas inibidores da ALS, flucarbazone-sódico e sulfossulfurom, quando aspergidos no outono e na primavera para o controle de Lolium multiflorum em $T$. aestivum demonstraram mais danos a $T$. aestivum em condições de temperatura mais elevada (primavera), enquanto o controle de L. multiflorum se mostrou dependente do herbicida e das épocas de aplicação. Foi verificada maior eficácia de flucarbazone-sódico no outono e de sulfossulfurom na primavera (HOSKINS et al., 2005). No entanto, quando sulfossulfurom foi utilizado em Agrotis stolonifera sob três condições de temperatura $\left(15,20\right.$ e $25^{\circ} \mathrm{C}$ ) ocorreram maiores percentuais de clorose e redução de peso nas plantas a $15^{\circ} \mathrm{C}$ (MCCULLOUGH e HART, 2008). Situação contrária ao teste com flucarbazone-sódico em Lolium perenne, sendo verificada elevada tolerância das plantas em temperaturas entre $10 \mathrm{e}$ $20^{\circ} \mathrm{C}$ em contraste aos resultados observados à temperatura de $30^{\circ} \mathrm{C}$ (MCCULLOUGH et al., 2012).

O efeito da temperatura na detoxificação dos herbicidas, a qual reduz a sua atividade, pode ser demonstrado mediante trabalho com rimsulfurom na cultura de milho (Zea mays L.). O incremento da temperatura elevou a detoxificação do herbicida até a obtenção da máxima seletividade para a cultura com temperaturas de $25-30^{\circ} \mathrm{C}$ e reduzida a $10^{\circ} \mathrm{C}$ (KOEPPE et al., 2000).

Os herbicidas bispiribaque-sódico e sulfossulfurom apresentaram efeitos contrastantes em relação às condições ótimas de temperatura para o aumento de atividade em Agrotis stolonifera. Bispiribaque-sódico foi mais efetivo na faixa de $20-30^{\circ} \mathrm{C}$, enquanto que sulfossulfurom se mostrou mais eficaz em baixas temperaturas $\left(15^{\circ} \mathrm{C}\right)$ (MCCULLOUGH e HART, 2006). Outro exemplo do efeito da interação entre herbicida e temperatura pode ser evidenciado com o uso de flumetsulam e metosulam no controle de Raphanus raphanistrum em seis condições de temperatura $(1,5,6,10$, 13 e $20^{\circ} \mathrm{C}$ ). Ambos os herbicidas apresentaram aumento de atividade proporcional à temperatura, sendo a eficácia $\left(E D_{50}\right)$ do flumetsulam incrementada com a elevação da temperatura em pelo menos seis vezes e a do metosulam menos de duas vezes (MADAFIGLIO et al., 2000). Em termos práticos, esses resultados implicam que sob condições de baixa temperatura seriam necessárias maiores doses de metosulam do que flumetsulam para o controle de $R$. raphanistrum (MADAFIGLIO et al., 2000).

\section{EFEITO DA UMIDADE RELATIVA DO AR (URA) SOBRE A EFICÁCIA DOS INIBIDORES DA ALS}

Preconiza-se que o aumento da umidade relativa do ar (URA) favoreça a atividade dos herbicidas inibidores da ALS no controle das plantas daninhas (KENT, WILLS e SHAW, 1991; LYM, 1992; HAGER et al., 1999; FUENTES e LEROUX, 2002; KIELOCH e DOMARADZKI, 2009; KIELOCH e KUCHARSKI, 2012). Por exemplo, trabalhos com ${ }^{14} \mathrm{C}$-imazetapir (inibidor da ALS com carbono marcado) evidenciaram maior absorção e translocação do herbicida em Ipomoea lacunosa com a elevação da umidade relativa do ar de 40 para 100 \% (KENT, WILLS e SHAW, 1991). Observaramse incrementos de $40 \%$ na absorção e de $4 \%$ na translocação de ${ }^{14} \mathrm{C}$-sulfometurom em Euphorbia esula mantida sob URA de 90-95 \%, contrastada à condição de URA de 20-30 \% (LYM, 1992). Todavia, a absorção e translocação de ${ }^{14} \mathrm{C}$-imazetapir em Ambrosia artemisiifolia foram similares sob URA de 65 e 85 \% (HAGER et al., 1999). 
Experimento realizado com híbridos de milho (Zea mays L.) para analisar o nível de tolerância ao herbicida rimsulfurom mostrou maior intoxicação da cultura com o herbicida aspergido a $75 \%$ de umidade relativa do ar, independentemente da temperatura, quando comparada a condição de $60 \%$ (FUENTES e LEROUX, 2002). Estudando o efeito da mistura dos herbicidas inibidores da ALS, iodossulfurom-metílico+mesossulfurom-metílico, sobre Alopecurus myosuroides Huds. foi obtida maior redução da biomassa com o aumento da umidade relativa do ar de $45 \%$ para $70 \%$ (KIELOCH e DOMARADZKI, 2009). Kieloch e Kucharski (2012) realizaram experimento similar com mistura de iodossulfurom-metílico+amidossulfurom em duas formulações, grânulos dispersíveis em água (WG) e dispersão em óleo (OD), no controle de Anthemis arvensis L., Galium aparine L. e Thlaspi arvense L. Não observaram redução do peso de A. arvensis L. e T. arvense L. entre $40 \mathrm{e}$ $70 \%$ de umidade relativa do ar. Entretanto, G. aparine L. apresentou maior redução de peso sob $70 \%$ do que sob $40 \%$ de umidade relativa do ar para as duas formulações WG e OD. Além disto, o mesmo estudo evidenciou que as formulações de herbicida OD apresentam maior eficiência do que as WG em condições de baixa umidade (40\%).

Dentre os fatores atribuídos ao aumento da eficácia dos herbicidas inibidores da ALS com a elevação da umidade relativa do ar pode-se destacar: permanência do herbicida em solução, redução da viscosidade e deposição de cera cuticular. Tais fatores em conjunto aumentam a possibilidade de entrada do produto através das folhas e posterior mobilidade na planta, que por consequência eleva o controle (COOK, BABIKER e DUNCAN, 1977; RAMSEY, STEPHENSON e HALL, 2005; STEPTOE, 2005). A alta umidade relativa do ar favorece a permanência do herbicida em solução, evitando a cristalização que poderia reduzir a absorção na superfície das folhas, o que aumenta a duração das gotas pela redução da pressão de vapor (COOK, BABIKER e DUNCAN, 1977). Outros fatores atribuídos à URA elevada envolvem a reduzida viscosidade da cera cuticular e maior hidratação da superfície foliar, afetando principalmente a atividade dos compostos lipídicos. Nesse processo, a mudança de viscosidade isoladamente não é suficiente para promover o aumento de difusão do herbicida, sendo necessário o aumento da umidade relativa do ar para que haja a hidratação da cutícula e viabilização do processo de difusão. Isso favorece a penetração de substâncias hidrofílicas na planta (RAMSEY, STEPHENSON e HALL, 2005).

A exposição de plantas a condições de umidade elevada também reduz a deposição de cera na cutícula. Em trabalho realizado com Commelina benghalensis em três regimes de umidade no solo $(25,50$ e $100 \%)$ foi observada menor espessura das folhas nos tratamentos com maior umidade (100 \%) em relação a $25 \%$, consecutivamente, $0,30 \pm 0,07 \mu \mathrm{m}$ e 1,1 $\pm 0,26 \mu \mathrm{m}$. Também foram verificadas reduções no número de tricomas e na quantidade de cera nas folhas das plantas mantidas em condições de maior umidade (STEPTOE, 2005). A maior presença de cera na superfície foliar limita a taxa de entrada do herbicida na planta, funcionando como barreira física, principalmente a compostos hidrofílicos. Da mesma forma, maior quantidade de tricomas reduz a eficácia da deposição das gotículas do herbicida ao formarem bolsas de ar sob as gotículas, o que impede o contato com a superfície foliar (HUANGFU, SONG e QIANG, 2009). Assim, as condições de umidade que podem afetar a eficácia dos herbicidas inibidores da ALS não se restringiriam somente as variações presentes no momento da aspersão do produto, mas também as precedentes a essa operação.

\section{EFEITO DA INTENSIDADE LUMINOSA SOBRE A EFICÁCIA DOS INIBIDORES DA ALS}

A intensidade luminosa afeta a atividade dos herbicidas inibidores da enzima ALS nas plantas (XIE, HSIAO e QUICK 1996; CAMARGO et al., 2012; MACIEL et al., 2011), mas ainda não está claro se o efeito da irradiância é positivo ou negativo na sua eficácia. O mecanismo envolvido no impacto da irradiância na atividade dos inibidores da ALS também não está adequadamente esclarecido. 
O benefício do sombreamento na atividade herbicida pode ser exemplificado pelo estudo realizado com ${ }^{14} \mathrm{C}$-imazametabenz-metil em Avena fatua. Quando as plantas foram submetidas a duas condições de luz $\left(400\right.$ e $\left.120 \mu \mathrm{mol} \mathrm{m}^{-2} \mathrm{~s}^{-1}\right)$, correspondentes a 0 e $70 \%$ de sombreamento, a absorção e a eficácia do produto após $96 \mathrm{~h}$ da aspersão foram 4,9\% maiores sob $120 \mu \mathrm{mol} \mathrm{m}{ }^{-2} \mathrm{~s}^{-1} \mathrm{em}$ comparação a $400 \mu \mathrm{mol} \mathrm{m}^{-2} \mathrm{~s}^{-1}$ (XIE, HSIAO e, QUICK, 1996). Contrariamente, outros experimentos evidenciaram que a luminosidade favorece a atividade de alguns herbicidas inibidores da ALS. O trabalho com o herbicida ${ }^{14} \mathrm{C}$-imazetapir em plantas de arroz vermelho (Oryza ssp.) tolerantes ao herbicida, mantidas em condições de câmara de crescimento $30 / 25^{\circ} \mathrm{C}$ (dia/noite) sobre quatro regimes de luz $\left(1066,677,259 \mathrm{e} 106 \mu \mathrm{mol} \mathrm{m}^{-2} \mathrm{~s}^{-1}\right)$ gerados por sombreamentos $(0,30,70 \mathrm{e}$ $90 \%$ ) demonstrou absorção superior do herbicida nas duas maiores intensidades luminosas (677 e $1066 \mu \mathrm{mol} \mathrm{m}{ }^{-2} \mathrm{~s}^{-1}$ ) que nas condições de irradiância reduzida (106 e $259 \mu \mathrm{mol} \mathrm{m}^{-2} \mathrm{~s}^{-1}$ ) (CAMARGO et al., 2012). Resultados similares foram obtidos na avaliação do efeito de imazetapir em três espécies de Poaceaes (Cynodon dactylon, Zoysia japonica e Axonopus compressus) mantidas em duas condições de sombreamento (0 e 50 \%). A intoxicação causada por imazetapir nas plantas, de maneira geral, foi maior nas condições de menor sombreamento (0 \%) quando comparado ao maior sombreamento (50 \%) (MACIEL et al., 2011).

\section{EFEITO DO ESTADO NUTRICIONAL SOBRE A EFICÁCIA DOS INIBIDORES DA ALS}

Hipotetiza-se que elevadas concentrações de alguns nutrientes favoreceriam a translocação e a atividade dos inibidores da ALS em algumas espécies de plantas daninhas (DASTGHEIB, 1993; CATHCART, CHANDLER e SWANTON, 2004; KIM et al., 2006; BROSNAN et al., 2010; SØNDERSKOV, SWANTON e KUDSK, 2012). Por exemplo, estudo realizado com clorsulfurom (60 g i.a. ha-1) em cultivar de trigo (Triticum aestivum) sensível (Rongotea) ao produto e avaliando

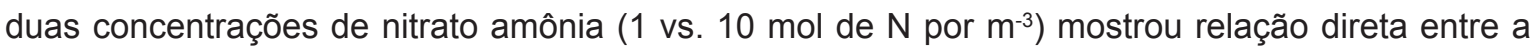
eficácia do herbicida e o nível de $\mathrm{N}$. Nas condições de maior concentração de nitrato de amônia (10 $\left.\mathrm{mol} \mathrm{m}^{-3}\right)$, o herbicida reduziu a massa das plantas em mais de $90 \%$ em relação ao tratamento sem herbicida. Todavia, sob baixos níveis de nitrato de amônia $\left(1 \mathrm{~mol} \mathrm{~m}^{-3}\right)$ não foram detectadas diferenças significativas na atividade do herbicida clorsulfurom (DASTGHEIB, 1993).

Nicossulfurom aspergido em Setaria viridis e Amaranthus retroflexus foi menos eficaz quando as plantas estavam sob níveis reduzidos de nitrato de amônia $(0,7 \mathrm{mM})$ em relação à condição de elevada $(7,7 \mathrm{mM})$ concentração do nutriente. De fato, para o controle de $50 \%$ das plantas de $\mathrm{S}$. viridis e A. retroflexus cultivadas em baixo teor de $\mathrm{N}$ houve a necessidade de doses de nicossulfurom seis vezes superiores àquela usada em plantas cultivadas sob elevada concentração do nutriente. Desse modo, maiores doses do herbicida seriam necessárias para o controle de ambas as plantas daninhas em solos com baixas quantidades de N (CATHCART, CHANDLER $e$ SWANTON, 2004).

Trabalho realizado com metsulfuron-metílico em Matricaria perforata Merat. e Papaver rhoeas L. usando seis níveis de nitrogênio $\left(0 ; 22,5 ; 45 ; 90 ; 180\right.$ e $\left.360 \mathrm{~kg} \mathrm{~N}^{-1}\right)$ evidenciou melhoria da curva de dose-resposta das plantas ao herbicida com o aumento do teor de nitrogênio. Também foi constatada redução da dose requerida para o controle de $50 \%$ das plantas daninhas $\left(\mathrm{ED}_{50}\right)$. Assim, para M. perforata cultivada com $0 \mathrm{~kg} \mathrm{ha}^{-1}$ de $\mathrm{N}$, os valores da $\mathrm{ED}_{50}$ foram reduzidos de $1,567 \mathrm{~g} \mathrm{ha}^{-1}$ de metsulfurom-metílico para $0,525 \mathrm{~g} \mathrm{ha}^{-1}$ quando o nível de $\mathrm{N}$ foi de $360 \mathrm{~kg} \mathrm{ha}^{-1}$. Similarmente, para P. rhoeas cultivada sob $0 \mathrm{~kg}^{-1} \mathrm{de} \mathrm{N}^{\mathrm{N}}$, os valores da $\mathrm{ED}_{50}$ passaram de $4,468 \mathrm{~g} \mathrm{ha}^{-1}$ de metsulfurommetílico para $0,87 \mathrm{~g} \mathrm{ha}^{-1}$ quando o nível de $\mathrm{N}$ foi de $360 \mathrm{~kg} \mathrm{ha}^{-1}$ (KIM et al., 2006).

Experimentos realizados com o herbicida flazassulfurom e a espécie Poa annua evidenciaram maior eficácia nos tratamentos que receberam duas aplicações de $75 \mathrm{~kg} \mathrm{~N}^{-1} \mathrm{em}$ contraste com os que receberam somente a aplicação do herbicida. No mesmo trabalho foram verificados incrementos de $18 \%$ na translocação do ${ }^{14} \mathrm{C}$-flazassulfurom da área tratada para outros 
tecidos da parte aérea da planta após $1 \mathrm{~h}$ e $22 \%$ após $4 \mathrm{~h}$ em comparação com as plantas tratadas com herbicidas, mas sem adubação nitrogenada (BROSNAN et al., 2010).

Experimento com tribenurom-metílico em Chenopodium album L., Tripleurospermun inodorum (L.) Schultz Bip. e Anagallis arvensis L. em quatro condições de nitrogênio (0; 20; 80; $120 \mathrm{~kg} \mathrm{~N} \mathrm{ha}^{-1}$ ) demonstrou elevada eficiência do herbicida em função do nível de N. Para T. inodorum, o aumento de $\mathrm{N}$ provocou redução da $\mathrm{ED}_{50}$ de 0,24 para $0,14 \mathrm{~g}$ i.a. ha- ${ }^{-1}$ com a mudança de 0 para $120 \mathrm{~kg} \mathrm{~N} \mathrm{ha}^{-1}$. Em A. arvensis também foi constatada redução de $\mathrm{ED}_{50}$ em resposta a elevação do teor de N, com decréscimo de 0,75 para 0,56 g i.a. ha-1 nas condições de 20 e $120 \mathrm{~kg} \mathrm{~N}^{-1}$ (SØNDERSKOV, SWANTON e KUDSK, 2012).

\section{INFLUÊNCIA DE ENZIMAS VEGETAIS NA AÇÃO DOS HERBICIDAS INIBIDORES DA ALS}

Existem dois grandes grupos de enzimas vegetais que podem afetar a atividade de herbicidas inibidores da ALS. No primeiro estão as enzimas envolvidas na alteração da molécula ativa e, portanto, causam a detoxificação dos herbicidas. No segundo grupo estão as enzimas que aliviam os efeitos iniciais dos herbicidas, principalmente os decorrentes do estresse oxidativo.

\subsection{ENZIMAS DETOXIFICADORAS DOS HERBICIDAS}

A detoxificação dos herbicidas pelas plantas impacta na seletividade para as culturas e plantas daninhas porque inativa o produto químico (VIDAL, 2002; COBB e READE, 2010). A tolerância conferida por detoxificação de herbicidas está geralmente associada a famílias de enzimas citocromo P450 monooxigenases (P450) e glutationa-S-transferases (GST) (READE, MILNER e COBB, 2004). As P450, uma das maiores famílias de enzimas, são encontradas na maioria dos organismos e estão localizadas no retículo endoplasmático e em alguns casos na membrana dos plastídios. A hidroxilação e a desalquilação constituem o principal mecanismo de desativação dos herbicidas catalisados por essas enzimas (POWLES e YU, 2010). Na reação são utilizados elétrons do NADPH para ativar oxigênio por uma enzima associada, citocromo P450 redutase. Um átomo do oxigênio molecular é incorporado no substrato (herbicida), enquanto o outro é reduzido para formar água (COBB e READE, 2010). Geralmente, os compostos formados são menos ativos do que a molécula original. Após a reação de hidroxilação ou desalquilação, normalmente, os produtos são glicosilados pela ação da UDP-difosfoglicose no complexo de Golgi e posteriormente transportados para o vacúolo pela ação das enzimas glicosil-transferases (GT) (COLEMAN, BLAKE-KALFF e DAVIES, 1997).

GST são enzimas que catalisam a conjugação do tripeptídeo glutationa (GSH) para vários substratos hidrofóbicos e geralmente citotóxicos (MARRS, 1996). As GST no citosol das células ativam as GSH, as quais conjugam os substratos tóxicos (herbicida entre outros compostos endógenos e exógenos) convertendo-os a produtos S-glutationilados. Esses são rapidamente transportados do citosol para dentro do vacúolo para posteriormente serem processados através da ação de transportadores específicos da classe ATP-ligantes cassete (EDWARDS, DIXON e WALBOT, 2000). As enzimas GST podem ser induzidas por estresses bióticos como infecções ou abióticos, tais como o estresse osmótico e temperaturas elevadas, além de estresses oxidativos ocasionados por herbicidas (DIXON, LAPTHORN e EDWARDS, 2002).

Há evidências de que a detoxificação de vários herbicidas inibidores da ALS esteja mais associada à atividade das enzimas P450 (KOEPPE et al., 2000; HIDAYAT e PRESTON, 2001; DENG e HATZIOS, 2003; YU et al., 2004; LIU et al., 2012) e, em menor grau, as GST (OZTETIK, 2010; WEI et al., 2010). Por exemplo, trabalho realizado com ${ }^{14} \mathrm{C}$-rimsulfurom em Zea mays L. empregando 
dois inibidores de P450, piperonil butóxido (PBO) e o inseticida organofosforado terbufós, sugere o envolvimento da detoxificação. De fato, a meia-vida do rimsulfurom nas folhas aumentou de $1,5 \mathrm{~h}$ (plantas não tratadas) para $16 \mathrm{~h}$ nas folhas das plantas tratadas com terbufós e em torno de $4,3 \mathrm{~h}$ para $\mathrm{PBO}$. A constatação de que havia detoxificação do rimsulfurom foi documentada mediante cromatografia (HPLC), sendo demonstrado que a primeira rota de detoxificação desse herbicida envolvia a hidroxilação do anel de pirimidina, seguido pela conjugação da glicose (glicosilados). Essa rota de detoxificação do herbicida constitui forte evidência do envolvimento de P450 no processo (KOEPPE et al., 2000).

O herbicida imazetapir aspergido em Digitaria sanguinalis (resistente ao produto), previamente tratada com inseticida malation (inibidor de P450), reduziu $\circ \mathrm{ED}_{50}$ de 102 para $34 \mathrm{~g} \mathrm{ha}^{-1}$ (HIDAYAT e PRESTON, 2001). De forma similar, a detoxificação de ${ }^{14} \mathrm{C}$-bensulfurommetílico em Oryza sativa L. cv. 'Lemont' foi reduzida em $40 \%$ quando o herbicida foi combinado ao inibidor de P450 (PBO) em comparação ao tratamento sem inibidor. Outros inibidores de P450 (1-aminobenzotriazole (ABT), citocromo c (CYT C), PBO e tetciclases (TET)) reduziram em mais de $60 \%$ a atividade da enzima de detoxificação. A principal reação de detoxificação do bensulfurommetílico obtida foi a desmetilação da molécula, catalizada por P450 (DENG e HATZIOS, 2003).

Estudo com Vulpia bromoides, espécie tolerante aos inibidores da ALS, empregando clorsulfurom e malation (inibidor de P450) evidenciou o envolvimento da detoxificação na insensibilidade da espécie ao herbicida. De fato, $\mathrm{a} \mathrm{ED}_{50}$ passou de $338 \mathrm{~g} \mathrm{ha}^{-1}$ na ausência do inibidor de detoxificação para $23 \mathrm{~g} \mathrm{ha}^{-1}$ na presença do inibidor (YU et al., 2004).

$\mathrm{Na}$ tentativa de elucidar o efeito de Cyt P450s na tolerância aos inibidores da ALS e verificar a possiblidade de transferência da característica para outras plantas, o gene CYP81A6 que codifica uma enzima P450, encontrado em arroz cultivado (Oryza sativa L. cv. 'Nipponbare'), foi inserido em Arabidopsis thaliana e Nicotiana tabacum. A confirmação da transferência e eficiência do referido gene após o processo de transformação foi obtida por meio da aspersão de bensulfurom-metílico na progênie de ambas as plantas, resultando em alto índice de sobrevivência das plantas transgênicas em comparação a plantas não transformadas (LIU et al., 2012).

A ação de GST em herbicidas inibidores da ALS foi demonstrada em experimento realizado com cultivares de trigo (Triticum aestivum L.) e cevada (Hordeum vulgare L.) aspergidas com tribenurom-metílico (seletivo para trigo e cevada). Nas raízes das cultivares de trigo Izgi-2001 e Alphu-200, tratadas com o herbicida, foram evidenciados aumentos na atividade de GST de mais de $180 \%$ em relação aos respectivos controles sem o herbicida. Consecutivamente, também foram observados incrementos superiores a $135 \%$ na atividade de GST na parte aérea das duas cultivares aspergidas em contraste às plantas não tratadas (OZTETIK, 2010). De forma análoga, quando o herbicida clorimurom-etílico foi utilizado em plantas de beterraba (Beta vulgaris) tolerantes

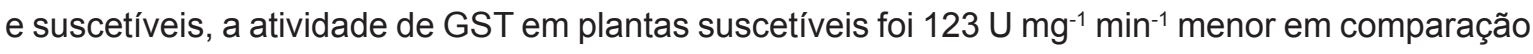
com as plantas tolerantes (WEl et al., 2010).

\subsection{ENZIMAS QUE REDUZEM O ESTRESSE OXIDATIVO NAS PLANTAS}

O estresse oxidativo resulta de condições que promovem a formação de espécies reativas de oxigênio (ROS) que danificam as membranas de organelas ou das células e, em consequência, causam a morte vegetal. Fatores ambientais que induzem a formação de ROS incluem a poluição do ar (aumento da quantidade de ozônio ou dióxido de enxofre), metais pesados, seca, calor, frio, danos mecânicos, luz (alta intensidade que conduz a fotoinibição), ataque de patógenos e herbicidas (BRAY, BAILEY-SERRES e WERETILNYK, 2000). O aumento nos níveis de ROS causam danos oxidativos a lipídios, proteínas e ao DNA, o que conduz a uma série de alterações nas propriedades das membranas como fluidez, transporte de íons, perda da atividade enzimática e inibição da síntese de proteínas, resultando na morte da célula (SHARMA et al., 2012). As ROS em plantas 
são produzidas nos cloroplastos, mitocôndrias e peroxissomos. A formação ocorre durante reações redox, incompleta redução do oxigênio ou oxidação da água, as quais produzem radicais de hidroxila $\left(\mathrm{HO}^{*}\right)$, superóxido $\left(\mathrm{O}_{2}^{-}\right)$e peróxido de hidrogênio $\left(\mathrm{H}_{2} \mathrm{O}_{2}\right)$ (BRAY, BAILEY-SERRES e WERETILNYK, 2000; APEL e HIRT, 2004; HEYNO et al., 2011).

As plantas apresentam alguns mecanismos que lhes permitem reduzir o efeito das ROS, convertendo-as em formas menos reativas. Tal processo depende da intensidade do estresse e da capacidade de produção de enzimas antioxidantes de cada espécie. As principais enzimas envolvidas na redução do estresse oxidativo são as superóxido dismutase (SOD), catalase (CAT), guaiacol peroxidase (GPX) e enzimas do ciclo ascorbato-glutationa (AsA-GSH), tais como ascorbato peroxidase (APX), monodehidroascorbato redutase (MDHAR), dehidroascorbato redutase (DHAR) e glutationa redutase (GR) (FOYER e NOCTOR, 2011; SHARMA et al., 2012).

Há poucas informações a respeito da formação de espécies reativas de oxigênio em herbicidas inibidores da ALS, principalmente correlação entre a inibição da síntese dos aminoácidos valina, leucina e isoleucina com estresse oxidativo (HASSAN e ALLA, 2005; ZABALZA et al., 2007). No entanto, alguns trabalhos evidenciaram o aumento dos níveis de determinadas enzimas antioxidantes em função da presença de herbicidas do grupo das sulfonilureias, mais especificamente de tribenurom-metílico e clorimurom-etílico (WANG e ZHOU, 2006; WANG et al., 2009; DUMAN et al., 2010).

\section{CONSIDERAÇÕES FINAIS}

Os herbicidas inibidores da ALS são produtos de ampla utilização na agricultura brasileira. Condições ambientais são determinantes da eficácia desses produtos. Da mesma forma, condições fisiológicas, principalmente o nível ou atividades de determinadas enzimas, impactam na eficácia dos inibidores da ALS e consequentemente na dose a ser recomendada.

Muitos trabalhos da literatura estudaram o efeito dos fatores isoladamente. Todavia, faltam trabalhos que estudem a interação dos fatores apresentados, principalmente aqueles realizados em condições de campo. Há necessidade de se investir em mais estudos integradores do conhecimento de forma a se poder desenvolver estratégias para otimizar a atividade dos herbicidas inibidores da ALS e possibilitar a redução da sua dose sem comprometer a eficácia no controle das infestantes e, por consequência, garantir a produtividade das lavouras.

\section{ABSTRACT \\ FACTORS THAT ENABLE THE REDUCTION OF THE RATE OF HERBICIDES INHIBITORS OF ALS ENZYME: A LITERATURE REVIEW}

The purpose of this review was to organize the data available on the literature regarding environmental and physiological factors that affect the efficacy of ALS inhibitors. Among environmental factors that affect the ALS inhibitors action are: temperature, relative humidity, irradiance and plant nutritional status. Among physiological factors that affect the herbicide dose are the enzymes involved on the detoxification of the herbicides and those enzymes involved on alleviating the oxidative stress due to herbicide action. Further integration knowledge studies are required in order to develop strategies to optimize the activity of ALS inhibitors allowing the reduction of herbicide dose without compromising the efficacy of weed control, and therefore ensure optimum crop yields.

KEY-WORDS: ENVIRONMENTAL VARIABLES; HERBICIDES; ENZYMES.

\section{REFERÊNCIAS}

1 APEL, K.; HIRT, H. Reactive oxygen species: metabolism, oxidative stress, and signal transduction. Annual Review of Plant Biology, Palo Alto, v.55, p. 373-399, 2004. 
2 BRASIL, Ministério da Agricultura, Pecuária e Abastecimento. Sistema de agrotóxicos fitossanitários (AGROFIT). Disponível em: <http://www.agricultura.gov.br/servicos-e-sistemas/sistemas/agrofit> Acesso: em 28 de julho de 2013.

3 BRAY, E. A.; BAILEY-SERRES, J.; WERETILNYK, E. Responses to abiotic stresses. In: BUCHANAN, B.; GRUISSEM, W.; JONES, R. Biochemistry \& molecular biology of plants. Rockville, MD, USA: American Society of Plant Biologists, 2000. p.1158-1202.

4 BROSNAN, J.T.; THOMS, A.W.; MCCULlOUGH, P. E.; ARMEL, G. R.; BREEDEN, G. K.; SOROCHAN, J. C.; MUELLER, T. C. Efficacy of flazasulfuron for control of annual bluegrass (Poa annua) and perennial ryegrass (Lolium perenne) as influenced by nitrogen. Weed Science, Lawrence, v.58, n.4, p.449-456, 2010.

5 CAMARGO, E.R.; SENSEMAN, S.A.; MCCAULEY, G.N.; BOWE, S.; HARDEN, J.; GUICE, J.B. Interaction between saflufenacil and imazethapyr in red rice (Oryza ssp.) and hemp sesbania (Sesbania exaltata) as affected by light intensity. Pest Management Science, Hoboken, v.68, n.7, p.1010-1018, 2012.

6 CARVALHO, L.B. de. Herbicidas. Lages-SC: Edição do Autor, 2013. 72 p.

7 CATHCART, R.J.; CHANDLER, K.; SWANTON, C.J. Fertilizer nitrogen rate and the response of weeds to herbicides. Weed Science, Lawrence, v.52, n.2, p.291-296, 2004.

8 COBB, A.H.; READE, J.P.H. Herbicides and plant physiology. $2^{\text {nd }}$ ed. Newport-UK: Harper Adams University, Wiley-Blackwell, 2010. 286 p.

9 COLEMAN, J.O.D.; BLAKE-KALFF, M.M.A.; DAVIES, T.G.E. Detoxification of xenobiotics by plants: chemical modification and vacuolar compartmentation. Trends in Plant Science, Oxford, v.2, n.4, p.144-151, 1997.

10 COOK, G.T.; BABIKER, A.G.T.; DUNCAN, H.J. Penetration of bean leaves by aminotriazole as influenced by adjuvants and humidity. Pesticide Science, Chichester, v.8, n.2, p.137-146, 1977.

11 DASTGHEIB, F. Response of wheat cultivars to chlorsulfuron and the effect of nitrogen availability. 1993. 257 p. Thesis (Doctored in Physiology). Lincoln University, New Zealand, 1993.

12 DENG, F.; HATZIOS, K.K. Characterization of cytochrome P450-mediated bensulfuron-methyl O-demethylation in rice. Pesticide Biochemistry and Physiology, San Diego, v. 74, n.2, p. 102-115, 2003.

13 DIXON, D.P.; LAPTHORN, A.; EDWARDS, R. Plant glutathione transferases. Genome Biology, Londres, v.3, n.3, p.1-10, 2002

14 DUMAN, F.; UREY, E.; TEMIZGUL, R.; BOZOK, F. Biological responses of a non-target aquatic plant (Nasturtium officinale) to the herbicide, tribenuron-methyl. Weed Biology and Management, Malden, v.10, n.2, p.81-90, 2010.

15 EDWARDS, R.; DIXON, D. P.; WALBOT, V. Plant glutathione S-transferases: enzymes with multiple functions in sickness and in health. Trends in Plant Science, Londres, v.5, n.5, p.193-198, 2000.

16 FOYER, C.H.; NOCTOR, G. Ascorbate and glutathione: the heart of the redox hub. Plant Physiology, Rockville, v.155, n.1, p.2-18, 2011.

17 FUENTES, C.L.; LEROUX, G.D. Effect of air temperature, relative humidity and growth stage on rimsulfurom tolerance in selected field maize hybrids. Agronomia Colombiana, Bogotá, v.20, n.3, p.21-30, 2002.

18 GEIER, P.W.; STAHLMAN, P.W.; HARGETT, J.G. Environmental and application effects on MON 37500 efficacy and phytotoxicity. Weed Science, Lawrence, v.47, n.6, p.736-739, 1999.

19 HAGER, A.G.; RENNER, K.A.; SCHABENBERGER, O.; PENNER, D. Soil moisture, relative humidity, and bentazon affect imazethapyr absorption and translocation in common ragweed (Ambrosia artemisiifolia). Weed Technology, Lawrence, v.13, n.2, p. 320-323, 1999.

20 HASSAN, N.M.; ALLA, M.M.N. Oxidative stress in herbicide-treated broad bean and maize plants. Acta Physiologiae Plantarum, Heidelberg, v.27, n.4, p.429-438, 2005. 
21 HEYNO, E.; MARY, V.; SCHOPFER, P.; KRIEGER-LISZKAY, A. Oxygen activation at the plasma membrane: relation between superoxide and hydroxyl radical production by isolated membranes. Planta, New York, v.234, n.1, p.35-45, 2011.

22 HEAP, I. The international survey of herbicide resistant weeds. Disponível em: <www.weedscience. org> Acesso em: 28 de julho de 2013.

23 HIDAYAT, I.; PRESTON, C. Cross-resistance to imazethapyr in a fluazifop-p-butyl-resistant population of Digitaria sanguinalis. Pesticide Biochemistry and Physiology, San Diego, v.71, n.3, p.190-195, 2001.

24 HOSKINS, A.J.; YOUNG, B.G.; KRAUSZ, R.F.; RUSSIN, J.S. Control of italian ryegrass (Lolium multiflorum) in winter wheat. Weed Technology, Lawrence, v.19, n.2, p.261-265, 2005.

25 HUANGFU, C.; SONG, X.; QIANG, S. Morphological disparities in the epidermal and anatomical features of the leaf among wild Brassica juncea populations. Weed Biology and Management, Malden, v.9, n.3, p.234-242, 2009.

26 JURSÍK, M.; SOUKUP, J.; HOLEC, J. Herbicide mode of actions and symptoms of plant injury by herbicides: inhibitors of acetolactate synthase (ALS inhibitors). Listy Cukrovarnické a Repařské, Praha-Czech Republic, v.126, n.11, p.376-379, 2010.

27 KENT, L.M.; WILLS, G.D.; SHAW, D.R. Influence of ammonium-sulfate, imazapyr, temperature, and relativehumidity on the absorption and translocation of imazethapyr. Weed Science, Lawrence, v.39, n.3, p. 412416, 1991.

$28 \mathrm{KIELOCH}, \mathrm{R} . ;$ DOMARADZKI, K. The effectiveness of two formulations of the mixture iodosulfuron methylsodium + mesosulfuron methyl in Apera spica-venti L. and Alopecurus myosuroides Huds: control depending on air temperature and humidity. In: INTERNATIONAL CONFERENCE ON WEED BIOLOGY, 13., Dijon. Annals... Dijon-França: AFPP, 2009. p.6.

$29 \mathrm{KIELOCH}$, R.; KUCHARSKI, M. Weed species response to two formulations of iodosulfuron methyl sodium and amidosulfuron mixture applied at various environmental conditions. Polish Journal of Agronomy, Puławy, v.8, n.8, p.15-19, 2012.

30 KIM, D.S.; MARSHALL, E.J.P.; CASELEY, J.C.; BRAIN, P. Modelling interactions between herbicide and nitrogen fertiliser in terms of weed response. Weed Research, Oxon, v.46, n.6, p.480-491, 2006.

31 KOEPPE, M.K.; HIRATA, C.M.; BROWN, W.H.; KENYON, W.H.; O'KEEFE, D.P.; LAU, S.C.; ZIMMERMAN, W.T.; GREEN, J.M. Basis of selectivity of the herbicide rimsulfuron in maize. Pesticide Biochemistry and Physiology, San Diego, v. 66, n.3, p.170-181, 2000.

32 LIU, C.; LIU, S.; WANG, F.; WANG, Y.; LIU, K. Expression of a rice CYP81A6 gene confers tolerance to bentazon and sulfonylurea herbicides in both Arabidopsis and tobacco. Plant Cell Tiss. Organ Cult. Dordrecht, v.109, n.3, p. 419-428, 2012.

33 LYM, R.G. Absorption and translocation of foliar-applied sulfometuron in leafy spurge (Euphorbia esula). Weed Science, Lawrence, v.40, n.3, p.477-481, 1992.

34 MACIEL, C.D.G.; POLETINE, J.P.; RAIMONDI, M.A.; RODRIGUES, M.; RIBEIRO, R.B.; COSTA, R.S.; MAIO, R.M.D. Desenvolvimento de gramados submetidos à aplicação de retardadores de crescimento em diferentes condições de luminosidade. Planta Daninha, Viçosa, v.29, n.2, p.383-395, 2011.

35 MADAFIGLIO, G.P.; MEDD, R.W.; CORNISH, P.S.; VAN DE VEN, R. Temperature-mediated responses of flumetsulam and metosulam on Raphanus raphanistrum. Weed Research, Oxon, v.40, n.4, p.387-395, 2000.

36 MARRS, K. A. The functions and regulation of glutathione s-transferases in plants. Annual Review of Plant Physiology and Plant Molecular Biology, Palo Alto, v.47, p. 127-158, 1996.

37 MCCULLOUGH, P.E.; HART, S.E. Temperature influences creeping bentgrass (Agrostis stolonifera) and annual bluegrass (Poa annua) response to bispyribac-sodium. Weed Technology, Lawrence, v.20, n.3, p.728-732, 2006.

38 MCCULLOUGH, P. E.; HART, S.E. Creeping bentgrass (Agrostis Stolonifera) tolerance to sulfosulfuron. 
Weed Technology, Lawrence, v.22, n.3, p.481-485, 2008.

39 MCCULLOUGH, P.E.; YU, J.; BROSNAN, J.T.; BREEDEN, G.K. Relative tolerance of perennial ryegrass (Lolium perenne) and tall fescue (Festuca arundinacea) to flucarbazone. Weed Technology, Lawrence, v.26, n.4, p.673-678, 2012.

40 OLSON, B.L.S.; AL-KHATIB, K.; STAHLMAN, P.; ISAKSON, P.J. Efficacy and metabolism of mon 37500 in Triticum aestivum and weedy grass species as affected by temperature and soil moisture. Weed Science, Lawrence, v.48, n.5, p.541-548, 2000.

41 OZTETIK, E. Effects of tribenuron-methyl treatment on glutathione S-transferase (GST) activities in some wheat and barley varieties. Pure and Applied Chemistry, North Carolina, v.82, n.1, p.289-297, 2010.

42 POLGE, N.D.; BARRETT, M. Temperature effects on imazaquin soil bioavailability, uptake, and metabolism in corn (Zea mays). Weed Science, Lawrence, v.45, n.2, p.198-204, 1997.

43 POWLES, S.B.; YU, Q. Evolution in action: plants resistant to herbicides. Annual Review of Plant Biology, Palo Alto, v.61, p.317-347, 2010.

44 RAMSEY, R.J.L.; STEPHENSON, G.R.; HALL, J.C. A review of the effects of humidity, humectants, and surfactant composition on the absorption and efficacy of highly water-soluble herbicides. Pesticide Biochemistry and Physiology, San Diego, v.82, n.2, p.162-175, 2005

45 READE, J.P.H.; MILNER, L.J.; COBB, A.H. A role for glutathione S-transferases in resistance to herbicides in grasses. Weed Science, Lawrence, v.52, n.3, p.468-474, 2004.

46 SHARMA, P.; JHA, A.B.; DUBEY, R.S.; PESSARAKLI, M. Reactive oxygen species, oxidative damage, and antioxidative defense mechanism in plants under stressful conditions. Journal of Botany, New York, v.2012, p.1-26, 2012.

47 SØNDERSKOV, M.; SWANTON, C.J.; KUDSK, P. Influence of nitrogen rate on the efficacy of herbicides with different modes of action. Weed Research, Malden, v.52, n.2, p.169-177, 2012.

48 STEPTOE, P.J. Influence of moisture stress on the herbicidal control of Commelina benghalensis. 2005. 60 p. Thesis (Master's degree in Science), University of Georgia, Athens, 2005.

49 TRANEL, P.; WRIGHT, T.R. Resistance of weeds to ALS-inhibiting herbicides: what have we learned?. Weed Science, Lawrence, v.50, n.6, p.700-712, 2002.

50 VIDAL, R.A. Ação dos herbicidas. Porto Alegre: Ribas Vidal, 2002. 89 p.

51 WANG, M.; ZHOU, Q. Effects of herbicide chlorimuron-ethyl on physiological mechanisms in wheat (Triticum aestivum). Ecotoxicology and Environmental Safety, v.64, p. 190-197, 2006.

52 WANG, M.; ZHOU, Q.; REN, L. Toxicological responses in wheat Triticum aestivum under jointstress of chlorimuron-ethyl and copper. Ecotoxicology and Environmental Safety, San Diego, v.72, n.2, p. 21212129,2009

53 WEI, D.; FENG-MING, M.A.; CHENG, Z.; TAO, B. Effect of chlorimuron-ethyl on biochemical mechanism in tolerant sugar beet. Agricultural Sciences in China, Oxon, v.9, n.12, p.1771-1776, 2010.

54 WILLINGHAM, S.D.; SENSEMAN, S.A.; MCCAULEY, G.N.; CHANDLER, J.M. Effect of temperature and propanil on penoxsulam efficacy, absorption, and translocation in alligatorweed (Alternanthera philoxeroides). Weed Science, Lawrence, v.56, n.6, p.780-784, 2008.

55 XIE, H.S.; HSIAO, A.I.; QUICK, W.A. Influence of temperature and light intensity on absorption, translocation and phytotoxicity of fenoxaprop-ethyl and imazamethabenz-methyl in Avena fatua. Journal of Plant Growth Regulation, New York, v.15, n.2, p. 57-62, 1996.

56 YU, Q.; FRIESEN, L.J.S.; ZHANG, X.Q.; POWLES, S.B. Tolerance to acetolactate synthase and acetylcoenzyme A carboxylase inhibiting herbicides in Vulpia bromoides is conferred by two co-existing resistance mechanisms. Pesticide Biochemistry and Physiology, San Diego, v.78, n.1, p.21-30, 2004. 
57 ZABALZA, A.; GASTON, S.; SANDALIO, L.M.; DEL RÍO, L.A.; ROYUELA, M. Oxidative stress is not related to the mode of action of herbicides that inhibit acetolactate synthase. Environmental and Experimental Botany, Oxford, v.59, n.2, p. 150-159, 2007.

\section{AGRADECIMENTOS}

Os autores agradecem o apoio da CAPES e do CNPQ. 